\title{
A STUDY ON THE MINIMUM REQUIREMENTS FOR THE ON-LINE, EFFICIENT AND ROBUST VALIDATION OF NEUTRON DETECTOR OPERATION AND MONITORING OF NEUTRON NOISE SIGNALS USING HARMONY THEORY NETWORKS ${ }^{1}$
}

\author{
Tatiana Tambouratzis ${ }^{\mathrm{a}}$, Laurent Pantera ${ }^{\mathrm{b}}$ and Petr Stulik ${ }^{\mathrm{c}}$ \\ a Department of Industrial Management \& Technology, \\ University of Piraeus, Piraeus 185 34, Greece \\ bLaboratoire des Programmes Expérimentaux et des Essais en Sûreté, \\ CEA/DES/IRESNE/DER/SPESI/LP2E/, Cadarache, \\ F-13108 Saint-Paul-Lez-Durance, France \\ 'Diagnostics and Radiation Safety Department, ÚJV Řeža.s., \\ Hlavní 130, Řež, 25068 Husinec,Czech Republic
}

\begin{abstract}
On-line monitoring (OLM) of nuclear reactors (NRs) incorporates - among other priorities - the concurrent verification of $(i)$ valid operation of the NR neutron detectors (NDs) and (ii) soundness of the captured neutron noise (NN) signals (NSs) per se. In this piece of research, efficient, timely, directly reconfigurable and non-invasive OLM is implemented for providing swift - yet precisedecisions upon the (i) identities of malfunctioning NDs and(ii) locations of NR instability/unexpected operation. The use of Harmony Theory Networks (HTNs)is put forward to this end, with the results demonstrating the ability of these constraint-satisfaction artificial neural networks (ANNs) to identify(a) the smallest possible set of NDs which, configured into $(b)$ the minimum number of 3-tuples of NDs operating on(c) the shortest NS time-window possible, instigate maximally efficient and accurate OLM. A proof-of-concept demonstration on the set of eight ex-core NDs and corresponding NSs of a simulated Pressurized Water nuclear Reactor $(P W R)$ exhibits $(i)$ significantly higher efficiency, at (ii) no detriment to localization accuracy, when employing only (iii) half of the original NDs and corresponding NSs, which are configured in (iv) a total of only two (out of the 56 combinatorially possible)3-tuples of NDs. Follow-up research shall investigate the scalability of the proposed methodology on the more extensive and homogeneous (i.e. "harder" in terms of ND/NS cardinality as well as of ranking/selection) dataset of the 36 in-core NSs of the same simulated NR.
\end{abstract}

\section{KEYWORDS}

Nuclear Reactor (NR),On-Line Monitoring (OLM), Neutron Noise (NN), Neutron Noise Signal (NS), Neutron Detector (ND), Computational Intelligence (CI),Artificial Neural Network (ANN),Harmony Theory Network (HTN), 3-tuple of NDs/NSs

${ }^{1}$ This piece of research is dedicated to the COVID-19 victims worldwide.

Dhinaharan Nagamalai et al. (Eds): CSEIT, WiMoNe, NCS, CIoT, CMLA, DMSE, NLPD - 2020

pp. 141-154, 2020. CS \& IT - CSCP 2020

DOI: $10.5121 /$ csit.2020.101113 


\section{ACKNOWLEDGEMENTS}

The research leading to these results (i) has received funding from the H2020 CORTEX Euratom Research and Training programme 2014-2018 under grant agreement No 754316, (ii) is supported by the Research Office of the University of Piraeus.

\section{LIST OF ACRONYMS}

\begin{tabular}{|l|l|}
\hline artificial neural network & ANN \\
\hline computational intelligence & CI \\
\hline cross-correlation & CC \\
\hline ex-core/out-of-core & ex- \\
\hline harmony theory network & HTN \\
\hline in-core & in- \\
\hline neutron detector & ND \\
\hline neutron noise & NN \\
\hline neutron (noise) signal & NS \\
\hline nuclear reactor & NR \\
\hline on-line monitoring & OLM \\
\hline pressurized-water NR & PWR \\
\hline principal component analysis & PCA \\
\hline simulated annealing & SA \\
\hline temperature & T \\
\hline
\end{tabular}

\section{INTRODUCTION}

\subsection{On-Line Monitoring of Nuclear Reactors}

Ever since the early installation and operation of nuclear reactors (NRs), on-line monitoring (OLM) has received special attention from nuclear engineering scientists, researchers as well as NR operators [1]. To date, OML covers the entire spectrum of safety

- from "operational", concerning the sustainable, controllable and maximally efficient chain-reaction with thermal neutrons from - and on - fissile material [2],

- to "radiation protection", concerning safety of the NR personnel at the local level, as well as ecological wellbeing of the flora, fauna and environment at the global level [3].

OLM of NRs encompasses the prompt processing and analysis of neutron noise (NN) signals(NSs) - as these are captured by NN detectors (NDs) -for ensuring the timely, noninvasive, consistent, reliable and (ideally) directly reconfigurable identification (as well as resolution) of various NR problems, including

(a) failing equipment (e.g. instrumentation, sensors, transmitters, NDs) and

(b) deviating-from-normal and/or inconsistent operation (e.g. aberrant coolant flow and/or temperature measurements).

Essential information on instrument calibration and verification, as well as on instrumentation/equipment/plant condition monitoring, can be found in [4-9]. Additionally, two relevant - complementary to one another, yet each comprehensive in its own focus and domain of interest - reviews of the literature on human/operational and computational intelligence (CI)based OLM appear in [10] and [11], respectively. 


\subsection{Organization of Presentation}

The remainder of this piece of research is organized as follows:

- Section 2 introduces the state-of-the-art relating to NR/OLM. A pertinent selection of innovative OLM approaches is presented, which (i)epitomizes the extensive range of preferred methodologies for tackling the multitude of OLM issues that may arise during NR operation and (ii) underscores the interplay between ND-/NS-derived information and custom-made OLM decisions/derivations/solutions. Subsequently, the motivation for the proposed methodology, as well as the advancement offered by the implemented encoding of the problem variables and constraints, is introduced in this Section.

- The NR set-up and characteristics of/dependencies between the out-of-coreNDs/NSs (exNDs/ex-NSs) of the dataset of [12] (which has been used for demonstrating the proposed OML approach) are detailed in Section 3.

- Section 4 provides a comprehensive introduction to the HarmonyTheory Network (HTN) [13] and its custom-made implementation for the concurrent selection of the minimal number of a) ex-NDs per se and b) 3-tuples [14] of the ex-NDs of a), which - combined with c) the shortest possible (of length 256) sliding time-window of the corresponding NSs - implement consistent, global, prompt and precise, non-invasive and directly reconfigurable OLM. Problem decomposition and gradual upscaling is implemented for endowing NR operation with computational (space- and time-) efficiency, at no compromise to the optimality of the returned ex-ND configuration/solution. The advantages of the proposed approach are further supported by a critical presentation of the obtained results.

- Section 5concludes the presentation by summarizing the main characteristics and innovation of the proposed approach, reporting on the importance of the findings, drawing keyconclusions and stating future extensions to the presented research.

\section{NuClear REACTORS \& ON-Line Monitoring}

\subsection{State-of-the-Art}

The extensive range of NR operation-related prerequisites and restrictions/controls/ constraints has resulted in (I) a multitude of nuclear protection guidelines, initiatives and standards,(II) primary information and reviews of the state-of-the-art on multi-sensor coordination (e.g. [1516]) as well as on modelling, estimation and control (e.g. [17]). Over the last decade, both research and development have delved into the implementational characteristics and properties that are necessary for rendering OLM consistently correct, maximally efficient, robust to missing - yet sensitive to erroneous- information, as well as capable of swift reconfiguration whenever deemed necessary.

A selective - yet representative - collection of pieces of research which employ, validate and co-ordinate sets of collaborating NDs (via the corresponding NSs) is provided next, with each implementation accompanied by a brief exposition of problem statement, execution and novelty:

- Principal component analysis (PCA) has been employed in [18] for mathematically modeling the relationships that hold between topologically related sets of self-powered NDs, culminating into an operational "detection \& isolation scheme" for four types of simulated faults (bias, drifting, precision degradation and complete failure). 
- A hybrid scheme, combining (i) Kalman filtering for estimating prompt neutron flux variations and(ii) the generalized likelihood ratio for detecting and diagnosing ND faults, has been tested successfully in [19], demonstrating robust on-line correction of the step change on simulated neutron flux data concurrently implementing moving control-rods and fluctuating power demands.

- Three (the observation, dependency and state) sub-models of a sensor model have been implemented in [20] for effectively co-ordinating, as well as integrating, competitive and/or disparate pieces of NS-derived information which include uncertainty in the ND observations.

- The combination of recurrent PCA and k-means clustering of NDs has been put forward in [21] for the consistent detection and severity evaluation of failing NDs.

- $\quad \mathrm{A} \mathrm{CI} /$ fuzzy-logic-based decentralized multi-sensor detection system with reduced energy demands [22] has been found successful in attaining a superior level of detection accuracy.

\subsection{Problem Statement \& Aims - Motivation for the Implemented Research - Problem Representation - Proposed Advancement in the State-of-the-Art}

The validation of (a) correct operation of the in- and ex-core NDs (in-NDs and ex-NDs, respectively) [1] and (b) soundness of the captured NSs (in-NSs and ex-NSs, respectively) per se, constitutes a prerequisite of successful OLM which is based on the agreement of the measured NSs with expected reference values ${ }^{2}$, salient characteristics of signal evolution and inter-signal comparisons. Further to rendering OLM completely automated as well as autonomous, an additional major motivation is the advancement of the state-of-the-art by also maximizing the time- and space-efficiency of OML, an endeavour that is instigated in this piece of research via the selection and subsequent utilization of

(i) the minimal set of NDs and

(ii) the minimum number of 3-tuples ${ }^{3}$ of "collaborating" NDs [14] (derived from the minimal set of(i)) which is required for consistently implementing OLM over (I) the entire NR, (II) the full spectrum of possible NR operating modes (e.g. footnote 2), (III) the extensive variety of coolant flow-regimes(e.g. bubbly, churn), and

(iii) the shortest sliding time-window that is capable of on-line (real-time) -yet consistent capture of the time-evolution of the NSs which pertain to the selected 3-tuples of NDs of (ii).

The concomitant satisfaction of (i)-(iii) exposes the minimal set(s) ${ }^{4}$ of NDs (and corresponding NSs) which is/are necessary - as well as sufficient - for accomplishing accurate, efficient as well as swift, non-invasive and directly reconfigurable NS-derived information processing and custom-made problem resolution over the entire NR and the full spectrum of possible NR operating/coolant flow-regime conditions (as described in point (ii) of this Section), with the optimum 3-tuple configurations of NDs/NSs being based on the current information acquired

\footnotetext{
${ }^{2}$ the ranges and trajectories of the signals are fully determined by NR construction (the NR transfer function) for each mode of operation (start-up, shut-down, stand-by, transient vs. steady-state etc.) as well as coolant flow-regime conditions

${ }^{3}$ it has been shown in [12] that 3-tuples of appropriately selected in-NDs are necessary as well as sufficient for the concurrent detection of erroneous in-NSs and/or malfunctioning in-NDs of the 3-tuple

${ }^{4}$ the NDs/NSs of these sets may well be distinct over different modes of NR operation as well as location of instability, in order to better capture the phenomena under development (also see footnote 2)
} 
directly, as well as exclusively, from the NSs in the form of CCs, on-line observed NS deviations, inter-signal comparisons etc.

\section{Data Characteristics and Problem Encoding}

\subsection{Dataset Description}

The data used for demonstrating the proposed approach constitutes the complete set of eight exNSs which have been collected by the corresponding ex-NDs of the pressurized-water NR (PWR) described in [12]. The special interest in ex-NSs - rather than in the 36 in-NSs of the same dataset which has been used for demonstrating the 3-tuple configuration of [14]-is based on the following criteria:

(a) on the one hand, the relatively small number of ex-NDs allows the implementation/evaluation of a proof-of-concept study concerning the application of the proposed approach to the entire set of ex-NDs of [12], which can then be transferred to other, more extensive, sets of in- as well as ex-NDs;

(b) on the other hand, the lack of the high frequency component ${ }^{5}$ between/across ex-NDs renders OLM significantly more challenging in terms of timeliness and validity of response, especially when compared to in-NDs during rapidly evolving phenomena [3];

(c) as a result of (b),the cross-correlation (CC) coefficients between ex-NSs (shown in Table 1) are significantly lower and more varied than those between the in-NSs of the same dataset, thus placing further demands as far as (i) the concurrent satisfaction of the combination of pertinent sources of NS information and (ii) the processing of considerably more requirements, are concerned;

(d) it is important to determine whether, how and with what gain in terms of computational complexity, the 3-tuple configuration can be applied - successfully as well as confidently to the full set of ex-NDs and ex-NSs, especially given the comparatively low CC-values between ex-NSs.

Table 1. The CC coefficients between the set of eight ex-NS of [12], revealing three clusters of "sufficiently" correlated - i.e. collaborating - NDs and demonstrating two overlapping parts between pairs of neighbouring clusters.

\begin{tabular}{|l|l|l|l|l|l|l|l|l|}
\hline ex-NS & 1 & 2 & 3 & 4 & 5 & 6 & 7 & 8 \\
\hline 1 & 1.0000 & 0.9974 & 0.9345 & 0.9353 & 0.5966 & 0.5926 & 0.5756 & 0.5654 \\
\hline 2 & 0.9974 & 1.0000 & 0.9530 & 0.9553 & 0.6435 & 0.6418 & 0.6233 & 0.6157 \\
\hline 3 & 0.9345 & 0.9530 & 1.0000 & 0.9989 & 0.8179 & 0.8132 & 0.7994 & 0.7886 \\
\hline 4 & 0.9353 & 0.9553 & 0.9989 & 1.0000 & 0.8174 & 0.8158 & 0.7992 & 0.7916 \\
\hline 5 & 0.5966 & 0.6435 & 0.8179 & 0.8174 & 1.0000 & 0.9973 & 0.9993 & 0.9956 \\
\hline 6 & 0.5926 & 0.6418 & 0.8132 & 0.8158 & 0.9973 & 1.0000 & 0.9969 & 0.9988 \\
\hline 7 & 0.5756 & 0.6233 & 0.7994 & 0.7992 & 0.9993 & 0.9969 & 1.0000 & 0.9970 \\
\hline 8 & 0.5654 & 0.6157 & 0.7886 & 0.7916 & 0.9956 & 0.9988 & 0.9970 & 1.0000 \\
\hline
\end{tabular}

5 “... the high frequency component of the neutron noise allows [facilitates] the detection of phenomena in the near vicinity of an in-core detector" [3] 


\subsection{Dataset Analysis - Clustering-Derived Assumptions and Problem-Dependent Constraints}

As can be observed in Table 1, the application of a "threshold" of 0.8 to the CC values between all the pairs of the eight ex-NSs reveals three pair-wise overlapping clusters of "sufficiently" correlated ex-NSs, with NS_cluster1, NS_cluster2 and NS_cluster3 comprising ex-NS1 to exNS4, ex-NS3 to ex-NS6 and ex- NS5 to ex-NS8 (coloured red, blue and green), respectively, as well as two overlapping areas (coloured darker shades of red and green, respectively).

In theory and, since all the pairs of ex-NSs belonging to the same cluster demonstrate similar behaviour, any such pair could be used for the consistent prediction of every other ex-NS of the same cluster. However, the criterion of "sufficiently high" CC values is not adequate per se, as explained in the following remark and accompanying example which demonstrates the importance of implementing ex-NS/ex-ND selection beyond values and thresholds alone, thereby further substantiating the rationale behind/necessity for the 3-tuple methodology:

- As can be derived from Table 1, the selection of ex-NS2 is "preferable"6 to that of ex-NS1 as the CC values of ex-NS2 with the remaining ex-NSs (ex-NS3 through to ex-NS8) are higher than those of ex-NS1, with the same observation holding for ex-NS4 over ex-NS3, ex-NS5 over ex-NS6 and ex-NS7 over ex-NS8.

- Even so, such an (purely CC-value based) selection of ex-ND2, ex-ND4, ex-ND5 and ex$\mathrm{ND}^{7}$ does not allow the implementation of valid ex-NS/ex-ND monitoring, as not all the ex-NDs of each of these 3-tuples belong to the same cluster, resulting innone of the exNS2, ex NS4, ex-NS5\} or \{ex-NS4, ex-NS5, ex-NS7\} 3-tuples satisfying the pairwise "CC $>0.8$ " requirement (Table 1 ).

\subsection{Problem Representation/Encoding}

According to the aforementioned criteria and requirements of sufficient CCs between ex-NSs for the purposes of OLM, only these 3-tuples of ex-NSs which demonstrate values exceeding 0.8 for all (three) of their pairwise-derived CCs are considered operational and - thus - only these are encoded in the present implementation.

Optimization is accomplished in a divide-and-conquer fashion, expressed as the joint utilization of the combination of the minimum possible:

- number of 3-tuples of "collaborating" ex-NDs in the manner demonstrated in [14],

- number of sufficiently (and, ideally, as highly as possible) correlated ex-NSs per se, which configured into 3-tuples of NDs/NSs - are capable of monitoring the entire area of interest and

- time-step of 1 which, combined with a statistically adequate time-window (of 256 for the present dataset), guarantees the on-line, efficient and robust concurrent identification of exND- (as well as of ex-NN/ex-NS-) related NR faults.

Problem decomposition and gradual upscaling is implemented for endowing NR operation with computational (both space- and time-) efficiency, at no compromise to the optimality of the returned ex-ND configuration/solution.

\footnotetext{
${ }^{6}$ in the sense that it is more highly correlated with the proximal ex-NSs and, thus, more appropriate for performing exNS/ex-ND validity checks at the vicinity of the corresponding 3-tuples

${ }^{7}$ as the ex-NDs corresponding to the ex-NSs with the highest correlations with the other NSs
} 


\section{Harmony Theory network Construction/Problem Encoding FOR THE IMPLEMENTATION OF ON-LINE MONITORING}

\subsection{Harmony Theory Networks}

The HTN [13] constitutes a semantically constructed ${ }^{8}$ two-layer artificial neural network (ANN) architecture which is adept at optimising under constraints (e.g. [23-24]). Instead of training, the problem is mapped directly to the nodes and connections of the HTN during construction in such a manner that the (semantic, problem-specific) collective compatibility of the activation values between connected nodes of the two layers quantitatively expresses the degree of "harmony"/fitness" of the HTN state, as this is calculated based on the satisfaction of the constraints dictated by the problem/data per se and encoded in the HTN connections between the two HTN layers. The main characteristics of HTN construction and operation for OLM are briefly defined here:

- The lower HTN layer comprises the "representational feature" nodes (RFs), which encode the problem-related information at the desired/appropriate level of description/ encoding. In the present case, each RF stands for a 3-tuple of NDs which collects information (a) from the corresponding 3-tuple of NSs, as well as (b) from every other "sufficiently" $(>0.8)$ correlated ${ }^{10} \mathrm{NS}$ of the same cluster(s) as the given NSs of the 3-tuple, in order to determine the expected/anticipated values and trajectories of as many NSs as possible and, consequently, to be able to further provide reliable decisions upon normal operation of the corresponding NDs of the 3-tuple per se. Each RF can acquire one of two states, namely +1 (active) if the encoded 3-tuple of NDs reflects both valid ND operation and agreement in the evolution of the corresponding 3-tuple of NSs, or -1 (inactive) if either (or both) of valid ND operation and agreement in the evolution of the 3-tuple of encoded NSs cannot be established.

- The upper HTN layer comprises the knowledge atoms (KAs), with each KA encoding a NS of the present dataset and acquiring one of two states, +1 (active) if the NS represented by the given KA can be consistently monitored by at least one 3-tuple of NDs encoded ina connected as well as currently active RF of the lower layer, and 0 if monitoring of the given NS cannot be established. Following HTN settling/convergence, the set of active KAs represents the maximal set of mutually compatible NSs that can be (i) successfully monitored by at least one 3-tuple of NDs and (ii) validated as far as anticipated/correct NR operation (encompassing both ND functionality and NS monitoring) is concerned.

- The HTN connections are - by construction - bidirectional, symmetric and strictly limited between RFs/NDs and KAs/NSs; additionally, for the problem-at-hand they are exclusively positive, with the weights of all the connections emanating from the same KA being normalized as well as equal to each other (as is customary in HTNs), thereby encoding the reinforcing relationships between connected RF/KA pairs via:

a) Direct monitoring of any given NS (encoded in a KA) by each RF which contains (in its 3tuple of encoded NDs) the ND corresponding to the encoded NS. This NS/ND relationship

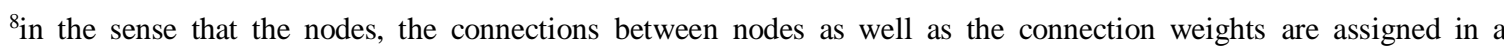
meaningful (expressive of the problem givens and constraints between givens), mathematical (predicate-logic\&normalization-based) manner

9i.e. compatibility between the activation values of connected HTN nodes of the two layers (as the HTN architecture does not allow within-layer connections)

${ }^{10}$ in which case, "sufficient" $(>0.8)$ CC with at least two of the NSs of the corresponding 3-tuple of NSs is required
} 
is implemented in the HTN via positive, reinforcing connections between the KAs and the relevant RFs (shown as fine black lines in Fig.1).

b) Indirect monitoring of any given NS (encoded in a KA) by each RF which does not contain (in its 3-tuple of encoded NDs) the ND corresponding to the encoded NS, yet contains at least two NDs that belong to the same cluster as the corresponding ND. This NS/ND relationship expresses the capability of the NDs of the 3-tuple to "indirectly" - yet securely - monitor and validate the given NS. Shown in Fig. 1 as bold red lines, these connections represent the transitive, "propagating" relationships between every KA and each connected RF that contains a 3-tuple of NDs that monitors at least two NSs from the same cluster as the given NS.

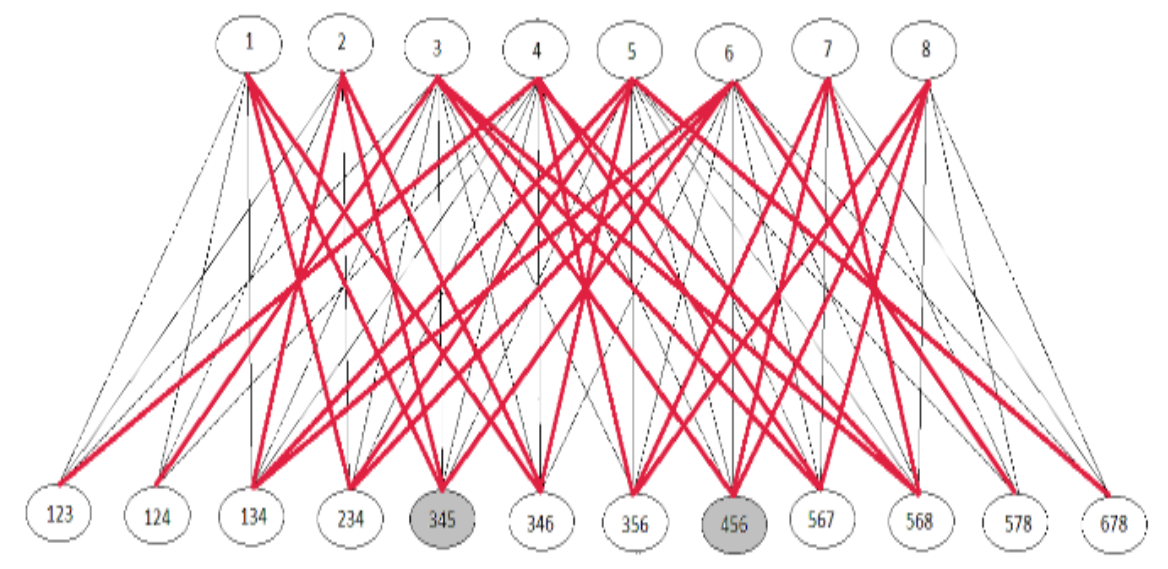

Fig. 1. HTN encoding of the (a) 12 sufficiently correlated (>0.8) ND 3-tuples in the RFs of the lower layer, (b) eight captured NSs in the KAs of the upper layer, (c) compatibility between the 3-tuples of sufficiently correlated NSs which can be used for deciding upon expected values and shapes of the NSs

The fine black lines (representing direct monitoring) between the nodes of the two layers of the HTN show three connections for the "exterior" NSs (1, 2, 7 and 8, corresponding to KAs 1, 2, 7 and 8, respectively) and six for the "interior" NSs (NSs 3, 4, 5 and 6, corresponding to KAs 3 though to 6, respectively). On the other hand, the bold red lines (representing the indirect connections) amount to three and four for the "exterior" and "interior" NSs, respectively.

- The HTN fitness function quantitatively expresses the "quality" of each HTN state (set of activation values over the entire sets of RFs and KAs), which - for the problem-at-hand - is implemented at two levels of optimization:

a) The KA-based level quantitatively expresses the "quality" of each HTN state in terms of the number of active KAs, per se, thus expressing the degree/level of compatibility between the active nodes of the two layers, and conveying the collective ability of the set of active RFs (3-tuples of NDs) to monitor as well as to verify as many as possible (and, ideally, all) of the NSs;

b) The RF-based level: in case of "ties" in (a), i.e. if more HTN states than one exist with the same maximum number of active KAs, the HTN state with the smallest number of active RFs is selected. Furthermore, if the same total number of 3-tuples of NDs is employed by more than one "best" solutions, the total number of the NDs per se (which are encoded in the active RFs of these solutions) are compared and the HTN state representing the smallest 
possible number of (i) 3-tuples of NDs as well as (ii) NDs per se, is selected; in the highly unlikely case where more than one such solutions co-occur, thresholding based on the lowest CC value between NSs belonging to the tied solutions is implemented for selecting the optimal configuration of NDs and 3-tuples of NDs.

The HTN operation characteristics for the present problem include (i) the elementary advancement (by 1) of the sliding time-window along the data and, consequently, on the timewindow employed for the calculation of the CC matrix, thus guaranteeing timely and robust - yet still sensitive - responses to changes within the NR (including changes in the NSs and/or the NDs per se); (ii) the application of simulated annealing (SA) [25] to the HTN, employed at each instance of the sliding time-window as follows. The HTN is initialized with the assignment of randomly assigned \pm 1 values to the RFs at an inaugural "high" value of the temperature parameter T of the HTN. The propagation of the assigned RF values to the connected KAs and the calculation of the total activations of the KAs is followed by thresholding for extracting the active KAs; this threshold is gradually raised (as T is lowered) during the SA process, such that one of the best - of maximal harmony - HTN state(s) is converged upon ${ }^{11}$. SA is appropriate for this task (and problem representation), as the occasional convergence of the HTN upon a suboptimum problem-state as the current solution for a given instance of the sliding time-window can be tolerated, as it is smoothed over/corrected by the previous and next HTN decisions. It should be mentioned, nonetheless, that a careful investigation and coordination of the HTN parameters and a conservative scheme of decrementing the T parameter during HTN settling is capable of minimizing premature, as well as imperfect, convergence to a non-optimum solution by initially considering the entire problem space and gradually focusing upon the more - and, eventually most - "promising" sections of the problem space, thus - as a rule - converging upon a state of maximal harmony.

\subsection{Harmony Theory Network Implementation via Step-Wise Optimization in Terms of Accuracy and Efficiency - Advancements, Advantages and Limitations}

The implementation of a serial (incremental) procedure of 3-tuples of NDs is not applicable to the present problem, as each sequentially selected next 3-tuple of NDs - even if "best" per se - far from guarantees the attainment of an optimal final solution. As, however, the implementation of combinatorial optimization does not necessarily scale up well (in terms of time complexity as well as of successful convergence) to problems of practical interest, an alternative problemdecomposition methodology involving a sequence of HTNs (at most as many as the monitored NSs) has been implemented and successfully tested for the problem-at-hand. The implemented HTNs may run either sequentially or in parallel (depending on the aim and/or time/spacecomputational complexity requirements/potential of the problem encoding), with each (the ith, $\mathrm{i}=1,2, \ldots, 8) \mathrm{HTN}$ (I) being set so as to allow for exactly i active RFs (i.e. $\mathrm{i}=1,2, \ldots, 8$ 3-tuples of NDs) for the maximization of $\mathrm{H}$ and (II) the SA settling procedure of each HTN, in turn, being automatically adapted according to this added constraint, thus configuring the activation values of the nodes of both layers such that the RFs that are converged upon by the HTN reveal the 3tuples of NDs which maximize $\mathrm{H}$ for the specific value of i. It is also important that such an implementation provides important information on the landscape of the problem space and, thus, on the interconnections and inter-constraints that apply between NSs (as well as between NDs) at different stages of OLM in terms of the numbers and identities of successfully monitored NSs by specific 3-tuples of NDs.

\footnotetext{
${ }^{11}$ in a probabilistic sense, where the probability of accepting an "inferior" HTN state during operation diminishes along with the drop in $\mathrm{T}$
} 
Table 2. A demonstration of HTN fitness of the optimal HTN-derived selection of NDs/NSs (highlighted 345-456 3-tuples) for successful OLM. All the alternative pairs of 3-tuples using the same ex-NDs/NSs (3, 4, 5 and 6) have been also tabulated for demonstrating the variation in HTN fitness that is reached by each such pair of 3-tuples, hence confirming HTN operation and settling to the HTN state of maximum $\mathrm{H}$ as well as highlighting the differences in $\mathrm{H}$ observed over the different combinations of pairs of 3-tuples.

\begin{tabular}{|c|c|c|c|c|c|c|}
\hline $\begin{array}{l}\text { pair of } \\
\text { CCs } \\
\text { ND/NS } \\
\text { between } \\
\text { 3-tuples of } \\
\text { NSs }\end{array}$ & $345-346$ & $345-356$ & 346-356 & $345-456$ & $346-456$ & $356-456$ \\
\hline 34 & $\begin{array}{l}0.9989 \\
(\times 2)\end{array}$ & 0.9989 & 0.9989 & 0.9989 & 0.9989 & - \\
\hline 35 & 0.8179 & $\begin{array}{l}0.8179 \\
(\times 2)\end{array}$ & 0.8179 & 0.8179 & - & 0.8179 \\
\hline 36 & 0.8132 & 0.8132 & $\begin{array}{l}0.8132 \\
(\times 2)\end{array}$ & - & 0.8132 & 0.8132 \\
\hline 45 & 0.8174 & 0.8174 & - & $\begin{array}{l}0.8174 \\
(\times 2)\end{array}$ & 0.8174 & 0.8174 \\
\hline 46 & 0.8158 & - & & 0.8158 & $\begin{array}{l}0.8158 \\
(\times 2)\end{array}$ & 0.8158 \\
\hline 56 & - & 0.9973 & 0.9973 & 0.9973 & 0.9973 & $\begin{array}{l}0.9973 \\
(\times 2)\end{array}$ \\
\hline $\begin{array}{l}\text { HTN } \\
\text { fitness } \epsilon[0 \\
1]\end{array}$ & 0.7174 & 0.7332 & 0.5336 & 0.7997 & 0.6001 & 0.6160 \\
\hline $\begin{array}{l}\text { occurrence } \\
(\%)\end{array}$ & 6.4 & 11.2 & - & 81.2 & - & 1.2 \\
\hline 250 trials & 16 & 28 & $\mathbf{0}$ & 203 & $\mathbf{0}$ & 3 \\
\hline
\end{tabular}

The settling procedure of the sequential HTN reported in this piece of research is described next. For the first iteration, the HTN SA-based "settling" procedure is initialized by imposing the activation value of +1 to a single, randomly selected, RF (i.e. a single 3-tuple of NDs of the lower level); the selected RF propagates its activation value to the connected KAs, the activation value of each KA is calculated and thresholded according to the binarization principle of HTN activation for the nodes of both HTN layers, and the entire set of activations is propagated back to the nodes of the lower layer, followed by roulette-wheel [26] selection of a single RF, which is assigned the activation value of 1 , and with all the other RFs being assigned activation values of 1 (inactive RFs). The process of limiting the number of active RFs to 1 is repeated according to the HTN settling procedure described in Section 4.1., whereby convergence upon (one of) the 3tuple(s) of NDs that monitor(s) the most NSs is achieved. If this number equals the total number of NSs, the procedure terminates and the 3-tuple of NDs is returned. Otherwise, this procedure is repeated from scratch, each time

- incrementing (by 1) the number of active 3-tuples of NDs (RFs) that are to be used concurrently for performing the second (or next) HTN iteration and

- $\quad$ retrieving the pair, triplet etc. (for the second, third iteration, etc., respectively) of 3-tuples of NDs of the lower HTN level which maximizes the number of active KAs (i.e. monitored NSs), 
up until all the NSs are monitored by the set of selected 3-tuples of NDs, in other words, all the KAs of the HTN are assigned activation values of +1 ) for the current number - and identity - of 3-tuples corresponding to the active RFs. The transparent construction of the HTN allows monitoring of the settling process as well as direct identification of (a) the 3-tuples of NDs (active RFs) and (b) NSs (active KAs) that optimise the computational (time- as well as space-) efficiency of OLM, where selection is based on the activations of the set of RFs prior to thresholding.
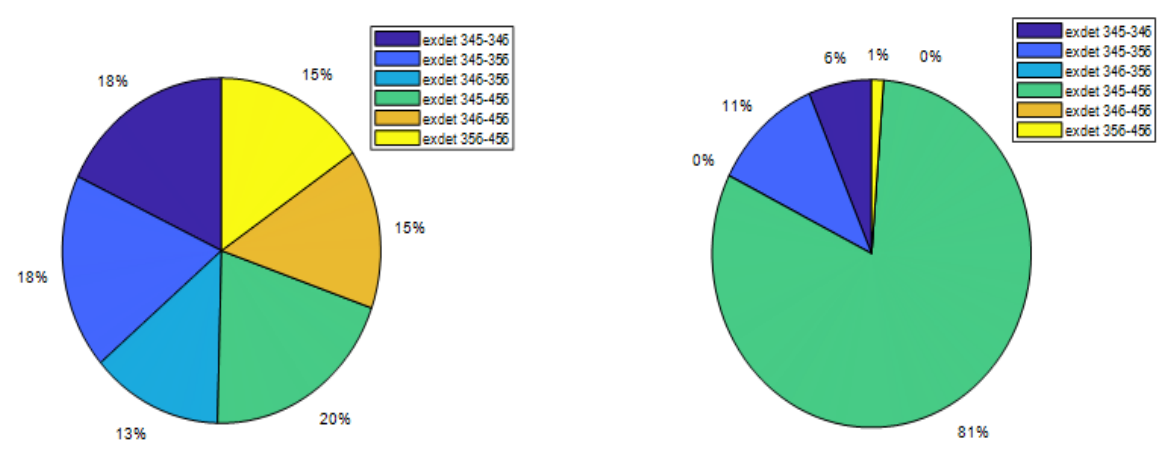

Fig. 2. Relative (\%) (a) HTN fitness and (b) frequency of HTN settling of the pairs of 3tuples of NDs/NSs comprising NDs/NSs 3, 4, 5 and 6 over 100 trials (shown in Table 2).

For the present dataset, the implementation and settling procedure of the HTN terminates after two operation/settling iterations: the 3-tuple selected at the first HTN/iteration is identified as \{exNS3, ex-NS4, ex-NS5 \}, which maximises the CC values between the selected NSs for time-step 1 and sliding time-window of 256. The second iteration/HTN converges upon the second 3-tuple of NSs, resulting in \{ex-NS3, ex-NS4, ex-NS5 $\}$ and \{ex-NS4, ex-NS5, ex-NS6\}). This configuration minimizes the number of involved NSs/NDs to 4 , while maximizing both the coverage over all eight NSs and the CCvalues within the two 3-tuples.

Table 2 presents a comparison of all the pairs of 3-tuples which contain the same NDs/NSs $(3,4$, 5and 6) as the optimal - as well as prevalent $(81.2 \%)$ - HTN solutions, i.e. are "neighbours" to the optimal k, i.e. in this case the 3-tuple of the first HTN is also included in the optimal configuration of 3-tuples of the second HTN solution. As can be seen, the second-best choice \{ex-NS3, ex-NS4, ex-NS5\} and \{ex-NS3, ex-NS5, ex-NS6\} has a slightly lower H (by 6.65\%), yet convergence upon this configuration of NDs/NSsis more than seven times less likely to occur than it is for the best configuration, thus further demonstrating the ability of the proposed HTN methodology to magnify - and, thus, distinguish between -small differences in HTN fitness. It is indicative that the identity of the selected NDs/NSs is the same in both configurations, with the superiority of the former pair of 3-tuples amounting to $0.0042 \times 2$ (normalized difference between the two sets of RF activation values).

In a complementary fashion, Fig. 2 illustrates the \% (relative) (a) HTN fitness and (b) frequency of HTN settling, of all the possible configurations of pairs of 3-tuples resulting from NDs/NSs 3, 4, 5 and6, revealing the magnification of $\mathrm{H}$ for small differences in CCs of pairs of involved NSs, with the optimal configuration being selected slightly more than 8 out of every 10 trials, and the remaining configurations appearing only occasionally, again in relation to their difference (in terms of $\mathrm{H}$ ) with the optimal solution. 


\section{CONCLuSions - Future Directions/EXTENSIONS}

The feasibility of creating an appropriately selected and fully operational minimal subset of sufficiently correlated ex-NDs employing the minimal time-window of 1 has been demonstrated on the set of eight ex-NDs and ex-NSs of [2]. Maximally efficient (with minimum response time) as well as accurate OLM of NRs is promoted, which is non-invasive and directly reconfigurable based on the characteristics and data-derived (e.g. statistical) properties of the NSs per se, encompassing (I) prompt detection of situations involving faulty NDs and/or NS anomalies, outliers or patterns indicating unexpected/abnormal operation etc. and (II) the accurate characterisation of NR irregularities in/deviations from expected operation. Moreover, the proposed methodology achieves the consistent (III) minimisation of the time- and spacecomplexity of OLM.

Further to a proof-of-concept, the results obtained from this investigation provide a directly implementable lower bound on the accuracy and consistency of operation of the proposed approach, especially in cases where (i) more NDs per se and/or (ii) more highly correlated NSs are available and/or (iii) longer time windows are implemented for the identification of phenomena of interest which evolve more gradually in time. It is also important that it remains possible - at any time - to (I) enrich the proposed OLM methodology with more NDs/NSs that are "sufficiently" correlated with the NS(s) of interest for expanding upon the initial findings derived from the reduced set of NDs, and/or to (II) exclude NDs/NSs which are redundant or have been detected as erroneous/faulty.

Future research shall focus upon fine-tuning the proposed implementation for such phenomena as transients, transitions between flow regimes and other occurrences during NR operation, as well as on whether the same (or a similar) configuration and implementation can be applied to the significantly larger, yet more highly correlated, set of 36 in-core NDs/NSs of the same dataset of [10], where the significantly higher CCs between in-NSs are compensated by the significantly larger number of (36)NDs/NSs to be simultaneously considered and optimised.

Demonstrating the feasibility of such an endeavour also paves the way for the hardware (H/W) implementation of maximally accurate as well as swift, automated decision-making upon the location(s) of NR instability and malfunctioning ND(s).

The entire computing/programming of the signal processing and HTN simulations has been implemented in the Matlab environment [27].

\section{REFERENCES}

[1] Eiler J., Glockler O., (2008). On-line monitoring for improving performance of nuclear power plants, Part 1, Instrument channel monitoring. Vienna, International Atomic Energy Agency, IAEANuclear Energy Series, ISSN 1995-7807; no. NP-T-1.1

[2] https://en.wikipedia.org/wiki/Fissile_material (retrieved April 21st, 2020)

[3] https://www.world-nuclear.org/information-library/safety-and-security/safety-of-plants/safety-ofnuclear-power-reactors.aspx

[4] https://www.energy.gov/sites/prod/files/2013/10/f4/QSR-RadiationProtection.pdf

[5] Hashemian, H.M. (2011). On-line monitoring applications in nuclear power plants. Progress inNuclear Energy 53, 167-181

[6] Behringer K., \& Crow R. (1980). Practical application of neutron noise analysis at boiling waterreactors, Wurenlingen, Switzerland. Swiss Federal Institute for Reactor Research Ch-5303 EIRBericht Nr 385

[7] Al Rashdan A., Smith J., St. Germain S., Ritter C., Agarwal V., Boring R., Ulrich T., \& Hansen J.(2018). Light water reactor sustainability program: development of a technology roadmap for 
onlinemonitoring of nuclear power plants INL/EXT-18-52206. U.S. Department of Energy, Office ofNuclear Energy

[8] https://www.energy.gov/sites/prod/files/2019/08/f65/ne-2019-advanced-sensors-instrumentationsummaries.pdf (retrieved April 21st, 2020)

[9] Pázsit I., \&Demazière C. (2010). Noise Techniques in Nuclear Systems, Handbook of NuclearEngineering, Cacuci D.G. (ed.), Springer ISBN: 978-0-387-98130-7, 1629-1737

[10] Ma J., \& Jiang J. (2011). Applications of fault detection and diagnosis methods in nuclear powerplants: a review, Progress in Nuclear Energy 53, 255-266

[11] Tambouratzis T., Giannatzis G., Kyriazis A., \&Siotropos P. (2020). Applying the computationalintelligence paradigm to nuclear power plant operation: a review (1990-2015), International Journalof Energy Optimization and Engineering 9, 27-109

[12] Chionis D., Dokhane H., Ferroukhi H., Girardin G., \&Pautz A. (2018). A PWR neutron noisephenomenology: part I - simulation of stochastic phenomena with SIMULATE 3K, Proceedings ofthe "PHYSOR 2018", IL, U.S.A.: American Nuclear Society, 1001-1012

[13] Smolensky P., (1986). Information processing in dynamical systems: Foundations of harmonytheory, Parallel distributed processing: Explorations in the microstructure of cognition, vol. 1:Foundation, ed. McClelland J.L., Rumelhart, D.E., MITPress/Bradford Books

[14] Tambouratzis T., Chionis D., \&Dokhane A., (2018). General regression neural networks for the concurrent, timely and reliable identification of detector malfunctions and/or nuclear reactor deviations from steady-state operation, in Proceedings of the 2018 IEEE Symposium Series on Computational Intelligence (SSCI), Bengaluru, India, November 18th-21st, 2018, 524-531

[15] Xiong N., \&Svensson P., (2002). Multi-sensor management for information fusion: issues andapproaches, Information Fusion 3, 163-186

[16] Li W.G., Wang Z., Wei G.L., Ma L.F., Hu J., \& Ding D., (2015). A survey on multisensor fusionand consensus filtering for sensor networks, Discrete Dynamics in Nature and Society, 1-15

[17] Maybeck S. (1982). Stochastic Models, Estimating, and Control. River Edge, NJ: Academic Press

[18] Peng X.J., Li Q., \&Wang K., (2015). Fault detection and isolation for self powered neutron detectorsbased on principal component analysis, Annals of Nuclear Energy 85, 213-219

[19] Sagar Y.V., Mishra A.K., Tiwari A.P., \&Degweker S.B., (2015). Online fault detection anddiagnosis of in-core neutron detectors using generalized likelihood ratio method, IEEE Transactionson Nuclear Science, 62, 3311-3323

[20] Durrant-Whyte, H.F., (2016). Sensor models and multisensor integration, The International Journalof Robotics Research, 7, 97-113

[21] Battacharyya A., Yogi V., Singla S., BhushanM., Kelkar M.G., Tiwari A.P., Pramanik M., \&BelurN., (2017). Adaptible, on-linemodels to detect and estimate gross error SPNDs, 2017 Indian ControlConference, January 4th-6th, 2017, 149-154

[22] Luo J.H., \& He X.T., (2018). A soft-hard combination decision fusion scheme for a clustereddistributed detection system with multiple sensors, E Sensors, 18, 4370 (doi:10.3390/s18124370)

[23] Tambouratzis T. (1998). A consensus-function artificial neural network for map colouring, IEEETransactions on Systems, Man, and Cybernetics, vol. 28, 721-728

[24] Tambouratzis T. (1999). A novel artificial neural network for sorting, IEEE Transactions onSystems, Man, and Cybernetics, vol. 29, 271-275

[25] Kirkpatrick S., Gelatt Jr C.D., \&Vecchi M.P. (1983). Optimization by simulated annealing, Science,vol. 220, 671-680

[26] Bäck T. (1996). Evolutionary Algorithms in Theory and Practice (1996), p. 120, Oxford Univ. Press

[27] MATLAB R2019.b, The MathWorks Inc. Natick, Massachusetts, U.S.A. 


\section{AUTHORS}

\section{Tatiana Tambouratzis}

BSc in Mathematics (University of Athens, Greece), MSc in Intelligent Systems and $\mathrm{PhD}$ in Artificial Neural Networks and Artificial Vision (Brunel University, U.K.). Researcher Grade IV, III, II (Institute of Informatics and Institute of Nuclear Technology - Radiation Protection, NCSR Demokritos, Attiki, Greece); Assistant and Associate Professor (Department of Industrial Management \& Technology, University of Piraeus, Piraeus, Greece); Visiting Researcher (Department of Nuclear Engineering, Chalmers University of Technology, Göteborg, Sweden).Scholarships from the Greek Scholarship Foundation, SERC award (U.K.), National Research Institute (Greece) and the Royal Society (U.K.), Fulbright Foundation (U.S.A.), Teaching Mobility Scholarship Program (U.S.A.). Fellow of the Institute of Mathematics and its Applications (IMA), membership no. 23668; member of the Institute of Electrical and Electronics Engineers (IEEE), membership no. 93994512. Member of the editorial advisory board of Progress in Nuclear Energy (area: Fission Technology).

\section{Laurent Pantera}

$\mathrm{PhD}$ in Heuristics and diagnostics for complex systems (University of Technology of Compiègne, UTC, France). Experimentalist at the CEA (French Alternative Energies and Atomic Energy Commission), he focused its activity on nuclear data processing and uncertainty calculation. He was in charge of the online gamma spectrometry measurements and experimental uncertainties assessment in the framework of the PHEBUS PF (Fission Products) international experimental programme to improve the understanding of the phenomena occurring during a severe accidents in a light water reactor. Afterwards, he joined the experimental CABRI International Programme (CIP) whose aim is to study the behavior of fuel rods at high burnup under Reactivity Initiated Accident (RIA).

\section{Petr Stulik}

Group Leader

Department of Diagnostics and Measurements

ÚJV Řež, a. s.

Hlavní 130, Řež

250 68, Husinec

Czech Republic

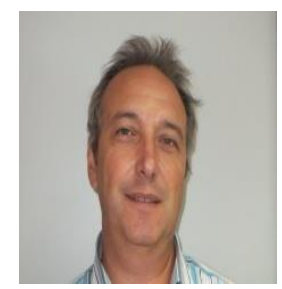

Disciplines: mechanics, experimental physics, nuclear physics

Skills \& expertise: mechanics, experimental physics, nuclear physics; pattern recognition, data analysis, data mining an knowledge discovery, statistical data analysis, data mining and knowledge discovery, statistical data analysis, information extraction Big Data, data processing, information filtering.

(C) 2020 By AIRCC Publishing Corporation. This article is published under the Creative Commons Attribution (CC BY) license 